\title{
Studien über die Veränderungen der Eiweisskörper und des kolloid=osmotischen Drucks im zu= und abströmenden Blutes der Niere.
}

I. Versuch an normalen sowie geschädigten Nieten.

\author{
Von \\ Kuranosuke Shida. \\ (志田藏之助) \\ (Aus der Medizinischen Klinik von Prof. Dr. T. Kato, \\ Tohoku-Reichsuniversität zu Sendai.)
}

Durch Untersuchungen von einer Reihe der Autoren ist dargetan worden, dass bei Nierenerkrankungen qualitative und quantitative Veränderungen des Bluteiweisses sowie eine Erniedrigung des kolloidosmotischen Drucks (k. o. D.) auftreten. An hiesiger Klinik hat Fukuhara ${ }^{1)}$ nachgewiesen, dass bei Tieren mit geschädigten Nieren nach Ausführung der Plasinaphäresis die Wiederherstellung des Bluteiweisses und dessen k. o. D. erheblich verzögert wird; San a d a ${ }^{2}$ hat bei Nierenläsion auch an Gewebseiweiss und dessen k. o. D. von der Norm auffallend abweichende Befunde erhoben; ferner haben Yasu$\mathrm{da}^{3)}$ und $\mathrm{K} \mathrm{usan \textrm {o } ^ { 4 } )}$ gefunden, dass die Eiweisskörper, die aus der Leber und den Muskeln in die Blutbahn mobilisiert werden, quantitativ und qualitativ verändert sind. Dennoch ist die Frage noch nicht endguiltig gelöst worden, ob derartige Veränderungen des Eiweisses und k. o. D. im Blut vorwiegend auf die durch Nierenschädigung sekundär hervorgerufenen Funktionsstörungen der Leber und anderer das Bluteiweiss regulierenden Organe zurückzuführen sei oder ob sie durch den Ausfall einer gewissen, im Zusammenhang mit der Regulation des Bluteiweisses stehenden Funktion, die der Niere selbst zukommen könnte, verursacht würden.

\footnotetext{
1) Fu ku hara, Tohoku Journ. Exp. Med., 1937, 30, 482.

2) Sa nad a, Ibid., 1936, 29, 156 ,

3) Y a s u d a, Ibid., 1937, 31, 456,

4) K u s a n o, Ibid., 1938, 34, 260.
} 
Die Niere beherrscht bekanntermassen nicht allein als das Ausscheidungsorgan der Endprodukte des Stoffwechsels die Harnbildung, sondern ihr kommen auch gewisse sonderbare Funktionen zu. Um einige Beispiele dafür anzuführen, schreiben Lascano-Ganzale $z^{5}{ }^{5}$ Asch off(f) u. a. der Niere die hämatopoetische Funktion zu; Nash $u$. Benedict, ${ }^{7)}$ Loeb, Atchly u. Benedict, ${ }^{8}$ Rabinowitsch, ${ }^{9}$ Gottlieb, ${ }^{10)}$ Thannhauser ${ }^{11)}$ u. a. haben die Fähigkeit zur Ammoniaksynthese in der normalen Niere und die Beeinträchtigung dieser Fähigkeit in erkrankter Niere nachgewiesen.

Um zur Frage, ob der Niere tatsächlich die regulatorische Funktion betreffend das Bluteiweiss und dessen k. o. D. zukommt, Stellung zu nehmen, muss man an dem der Niere zuströmenden und dem davon abströmenden Blut das Eiweissbild und das Verhalten des k. o. D. verfolgen.

In einschlägiger Literatur sind derartige Versuche nur von $\mathrm{W}$ i e $\mathrm{n}$ er, ${ }^{12)}$ Tanaka, ${ }^{13)}$ Pein, ${ }^{14)}$ Achard, ${ }^{15)}$ Häbler, Günther, Scheiner, Wagner u. Wörne ${ }^{16)}$ niedergelegt. Wi en e $r^{12)}$ hat nämlich bei Untersuchungen des Blutes aus Nierenvenen und Arterien von Hund gefunden, dass in der Norm im Nierenvenenblut der Gesamt- $\mathrm{N}$ herabgesetzt, hingegen der Globulin- $\mathrm{N}$ erhöht ist, wobei der Albumin-Globulin-Quotient niedrige Werte aufweist; daraus hat er Schluss gezogen, dass die Niere unter normalen Verhältnissen das Globulin an das Blut abgibt. Weiterhin hat er an 2 Uranhunden gleiche Bestimmungen ausgeführt und kam zum Ergebnis, dass trotzdem hierbei das Bluteiweiss in den Harn verloren geht, sowohl das Gesamteiweiss wie auch der Albumin-GlobulinQuotient im Venenblut gleichfalls erhöht war; er äusserte sich, dass diese Erklärung schwer sei. $\mathrm{Tan} \mathrm{a} \mathrm{k} \mathrm{a}{ }^{13)}$ hat in darauf gerichteten Versuchen konstatiert, dass der Gesamt-N im nüchternen Zustand im Nierenvenenblut höhere Werte auf weist, während er 2 Stunden nach der Nahrungsaufnahme hingegen im arteriellen Blut eher mehr vermehrt gefunden wird, ferner dass der Albumin-GlobulinQuotient und Rest-N nach dem Durchtritt des Blutes durch die Niere stets niedrigere Werte aufweisen. Der weitere, von ihm an mit Uran oder Kantharidin

5) La s can o-Ganzalez, Fol. Haemat., 1933, 50, 278.

6) Asch off, Schweiz. med. Wschr., 1935, 193.

7) Nash u. Benedict, Journ. Biol. Chem., 1918, 35, 101.

8) Loeb, Atchly u. Benedict, Ibid., 1924, 60, 491.

9) Rabinowits ch, Arch. Intern. Med., 1924, 33, 394.

10) Gottlie b, Biochem. Ztsehr., 1928, 194, 151.

11) Thannhauser, Klin. Wschr., 1933, 49.

12) Wiener, Ztschr. f. physiol. Chem., 1912, 82, 243.

13) Ta naka, Tokyo Iji Sinsi, 1928, 403.

14) Pein, Ztschr. f. d. ges. exp. Med., 1932, 82, 387.

15) A ch a rd, C. r. Soc. Biol., Paris, 1934, 116, 94.

16) Häbler, $G$ ünther, Scheiner, Wagner u. Wörner, Ztschr. f. d. ges. exp. Med., 1934, 94, 596. 
vergifteten Hunden ausgeführte Versuch fiel nicht eindeutig wie die Ergebnisse von Wi en e $\mathrm{r}^{\mathbf{1 2})}$ aus, indem der Gesamt-N und der Albumin-Globulin-Quotient bald im arteriellen, bald aber im venösen Blut höhere Werte aufwiesen. Pein ${ }^{14}$ ? führte an Nierenvenenblut des Hundes Bestimmungen nicht allein des Eiweisses, sondern auch des k. o. D. aus und kam zum Ergebnis, dass das Serumeiweiss im Nierenvenenblut unter gewöhnlichen Verhältnissen niedriger war und auch der k. o.D. niedrigere Werte aufzuweisen pflegte, und dass wenn bei Versuchstieren durch Injektionen von Salzlösung eine Senkung des k. o.D. des Blutes herbeigeführte wurde, oben erwähnte Verhältnisse sich ganz umgekehrt verhielten, indem das Eiweiss und der k. o.D. im Nierenvemenblut ohne Ausnahme, besonders aber der letztere eine deutliche Zunahme erfuhr. Aus diesem Ergebnis hat er die Vermutung ausgesprochen, dass die Niere auf den k. o. D. des Blut eine gewisse regulatorische Funktion ausüben müsse. A $\mathrm{chard}{ }^{(5)}$ hat im Versuch an gesunden Hunden nachgewiesen, dass die Gesamteiweissmenge des Blutes nach Nierenpassage mässig herabgesetzt ist; er ist der Meinung, dass diese Herabsetzung vorwiegend auf der Abnahme des Albumins beruhe. Häbler, Günther, $\mathrm{Scheiner}$, W agner und $\mathrm{W}$ örne $\mathrm{r}^{16)}$ fanden in einschlägigen Versuchen wie Pein, ${ }^{14)}$ dass der k. o. D. des Blutes meistens im Arterienblut, aber unter Umständen auch im Nierenvenenblut höhere Werte aufweisen könne. Im Hinblick auf die Tatsache, dass im letzteren Fall der k. o.D.des Arterienblutes relativ niedrig ist, sind sie der Meinung, dass hier der k.o.D. im Sinne der Kolloid-osmoregulation nach dem Durchtritt des Blutes durch die Niere eine Erhöhung erfahren dürfte.

Die Studien über das Verhalten des Bluteiweisses und des k. o. D. in dem die Niere durchspülenden Blut sind noch nicht hinreichend und haben, wie oben angeführt, noch kaum zu einheitlichem Resultat geführt; die Frage, welchen Einfluss die Niere, sich auf jeweilige verschiedene Zustandsänderungen des Organismus einstellend, auf die Eiweisskörper im die Niere durch spülenden Blute ausüben würde, ist fast noch im Dunkel gehïllt. An Kaninchen mit normalen sowie pathologischen Nieren habe ich Bestimmungen des Bluteiweisses und k. o. D. im der Niere zufliessenden und davon abfliessenden Blut ausgeführt und weiterhin auch die Beeinflussung dieser Grössen durch Aderlass und Bluttransfusion sowie die Veränderungen derselben durch Verabreichung von verschidenen Diureticis verfolgt. Ferner wurde darauf untersucht, ob und inwieweit in der Niere zirkulierendes Bluteiweiss und dessen k. o. D. bei Schädigung der Leber durch die Niere beeinflusst wird, weil dies bis dahin noch eine offene Frage bleibt, obwohl die Leber und die Nieren funktionell zueinander innig zusammenhängende Organe sind, vor allem aber für den Eiweisstoffwechsel in sehr intimer Beziehung stehen. 
In vorliegender Mitteilung seien Versuchsergebnisse, welche an normalen Kaninchen und an mit Uran oder Kantharidin vergifteten Kaninchen in dieser Richtung gewonnen wurden, angeführt.

\section{Versuchsmethode.}

Als Versuchsmaterial wurden gesunde Kaninchen von ungefähr $2 \mathrm{~kg}$ Körpergewicht verwandt. Am Versuchstag wurde das Versuchstier morgens nüchtern an den elektrisch angewärmten Tierhalter in der Rückenlage gefesselt, es wurde dann ihm eine 25\% ige Äthylurethanlösung in Mengen von $1,5 \mathrm{ccm}$ pro $\mathrm{kg}$ Körpergewicht subkutan injiziert. Nachdem das betreffende Tier dadurch ganz ruhig geworden war, wurde die Laparotomie ausgeführt, behufs Erleichterung der nachträglich vorzunehmenden Blutentnahme wurden linksseitige Nierenvenen abpräpariert, worauf die Bauchhöhle mit K och er's Klemme provisorisch verschlossen wurde. Demnächst wurde zwecks Entnahme arteriellen Blutes eine Kanüle in die A. carotis eingelegt. Nach Beendigung obiger Vorbereitungsmassregel liess man Versuchstiere zur Vermeidung der direkten Einflüsse der Operation ca. 2 Stunden lang ausruhen. Bei der Blutentnahme ging man so vor, dass man stets das arterielle Blut zuerst entnahm, im direkten Anschluss daran die Bauchhöhle wieder eröffnete und dann ohne Stauung mit einer durch trockne Hitze sterilisierten Spritze die V. renalis punktionierte und mit gleicher Geschwindigkeit wie der Blutstrom vorsichtig das venöse Blut entnahm. Die jedesmal entnommene Blutmenge betrug je ca. $5 \mathrm{ccm}$.

An so gewonnenen arteriellen und venösen Blutproben wurde das Serumeiweiss mit Pulfrich's Eintauchrefraktometer, der k. o. D. nach der Methode von Krogh u. Nakazaw a ${ }^{\text {(n) }}$ gemessen, ferner die Eiweissfraktionen mit dem von Parnus-Wagne ${ }^{13)}$ konstruierten Apparat nach der Methode von How ${ }^{19)}$ bestimmt. Der Hämoglobingehalt wurde mittels Fleischl-Miescher's Hämometers, das Serum-NaCl nach der R u s z n yá k'schen Methode bestimmt.

\section{Versuch an normalen Kaninchen.}

Es wurden 10 Versuche angestellt (Tab. 1).

Der Hämoglobingehalt zeigte zur Hälfte der Fälle (Vetsuch 1, 5, 6, 8 u. 9) im Nierenvenenblut und Arterienblut gleiche Werte, zur anderen Hälfte (Versuch 2, 3, 4, 7 u. 10) im erstern eine geringe Zunahme, deshalb kann man nichts einheitliches darüber aussagen. Da jedoch jedwede Abnahme in keinem Fall auftrat, ergab sich im Durchschnitt geringfügige Zunahme. Im Gegensatz hierzu, abgesehen von einem

17) Krogh u. Nakazawa, Biochem. Ztschr., 1927, 188, 241.

18) Parnus-Wagner, Ibid., 1921, 125, 253.

19) How e, Journ. Biol. Chem., 1921, 49, 93, 109 u. 115. 
Tabelle

Versuch an normalen

\begin{tabular}{|c|c|c|c|c|c|c|c|c|c|c|}
\hline \multirow{3}{*}{$\begin{array}{l}\text { Versuchs- } \\
\text { nummer u. } \\
\text { Geschlecht }\end{array}$} & \multirow{3}{*}{$\begin{array}{l}\text { Körper- } \\
\text { gewicht } \\
\quad \text { (kg) }\end{array}$} & \multicolumn{3}{|c|}{ Hämoglobin } & \multicolumn{3}{|c|}{ Serumeiweiss } & \multicolumn{3}{|c|}{ K. o. D. } \\
\hline & & \multicolumn{2}{|c|}{$\mathrm{g} / \mathrm{dl}$} & \multirow{2}{*}{${ }_{1}^{\frac{8}{x}}$} & \multicolumn{2}{|c|}{$\%$} & \multirow{2}{*}{$\begin{array}{c}8 \\
\frac{8}{x} \\
41 \\
1 \\
1\end{array}$} & \multicolumn{2}{|c|}{$\mathrm{mmH}_{2} \mathrm{O}$} & \multirow{2}{*}{$\begin{array}{c}8 \\
0 \\
x \\
4 \\
1 \\
1\end{array} \mid$} \\
\hline & & A & V & & $\mathbf{A}$ & V & & A & V & \\
\hline 1 古 & 1,89 & 15,08 & 15,08 & \pm 0 & 6,31 & 6,12 & $-3,0$ & 283 & 258 & $-8,8$ \\
\hline $2 \delta$ & 1,65 & 13,64 & 13,67 & $+0,2$ & 6,38 & 6,16 & $-3,4$ & 280 & 261 & $-6,8$ \\
\hline 3 ㅇ & 1,75 & 16,23 & 16,48 & $+1,5$ & 5,95 & 5,88 & $-1,2$ & 247 & 240 & $-2,8$ \\
\hline 4 우 & 1,86 & 14,52 & 14,58 & $+0,4$ & 6,85 & 6,85 & \pm 0 & 308 & 298 & $-3,2$ \\
\hline 5 今 & 2,40 & 14,80 & 14,80 & \pm 0 & 6,90 & 6,79 & $-1,6$ & 311 & 290 & $-6,7$ \\
\hline 6 告 & 1,93 & 14,52 & 14,52 & \pm 0 & 7,20 & 7,00 & $-2,8$ & 309 & 287 & $-7,1$ \\
\hline 7 ๙ & 2,18 & 14,52 & 14,66 & $+1,0$ & 6,14 & 6,08 & $-1,0$ & 285 & 269 & $-6,3$ \\
\hline 8 우 & 1,81 & 14,05 & 14,05 & \pm 0 & 6,32 & 6,23 & $-1,4$ & 287 & 275 & $-4,2$ \\
\hline $9 \delta$ & 2,10 & 14,24 & 14,24 & \pm 0 & 6,77 & 6,59 & $-2,7$ & 280 & 264 & $-5,7$ \\
\hline $10 \delta$ & 2,20 & 14,95 & 15,19 & $+1,6$ & 7,10 & 6,98 & $-1,7$ & 310 & 293 & $-5,5$ \\
\hline \multicolumn{2}{|c|}{ Maximum } & 16,23 & 16,48 & & 7,20 & 7,00 & & 311 & 298 & \\
\hline \multicolumn{2}{|c|}{ Minimum } & 13,64 & 13,67 & & 5,95 & 5,88 & & 247 & 240 & \\
\hline \multicolumn{2}{|c|}{ Durchschnitt } & 14,65 & 14,73 & $+0,5$ & 6,59 & 6,47 & $-1,9$ & 290 & 273 & $-5,7$ \\
\hline
\end{tabular}

A : Arterienblut.

V: Nierenvenenblut.

Ausnahmefall (Versuch 4), wo Serumeiweiss im Arterien- und Venenblut gleiche Werte aufwies, zeigte das Serumeiweiss im übrigen 9 Fällen in Nierenvenenblut niedrigere Werte, indem es gegenüber dem im Arterienblut im Mittel eine prozentische Abnahme um 1,9 (1,0-3,4) erfuhr. K. o. D. war nach dem Durchtritt des Blutes durch die Niere ohne Ausnahme erniedrigt; im venösen Blut trat nämlich eine $A b$ nahme von durchschnittlich 5,7\% (2,8-8,8\%). Da diese prozentische Erniedrigung von k. o.D. grösser als die des Serumeiweisses war, erfuhr der kolloid-osmotische Druck pro 1\% Eiweiss (unter kurz als Druck pro \% bezeichnet) im Nierenvenenblut ausschliesslich eine Abnahme, welche im Durchschnitt um 3,7\% (1,7-5,4\%) grösser war als im Arterienblut.

Was die Eiweissfraktionen anbelangt, erfuhren nach dem Durch- 
1.

Kaninchen.

\begin{tabular}{|c|c|c|c|c|c|c|c|c|c|c|}
\hline \multicolumn{3}{|c|}{ Druck pro\% } & \multicolumn{3}{|c|}{ Gesamt-N } & \multicolumn{3}{|c|}{ Rest-N } & \multirow{2}{*}{\multicolumn{2}{|c|}{$\frac{\text { Albumin-N }}{\mathrm{mg} / \mathrm{dl}}$}} \\
\hline \multicolumn{2}{|c|}{$\mathrm{mmH}_{2} \mathrm{O}$} & \multirow{2}{*}{$\begin{array}{l}8 \\
\frac{8}{x} \\
1 \\
1 \\
1\end{array}$} & \multicolumn{2}{|c|}{$\mathrm{mg} / \mathrm{dl}$} & \multirow{2}{*}{$\begin{array}{c}8 \\
8 \\
\times \\
x \\
1 \\
1\end{array}$} & \multicolumn{2}{|c|}{$\mathrm{mg} / \mathrm{dl}$} & \multirow{2}{*}{$\begin{array}{l}8 \\
\overline{1} \\
x \\
1 \\
1\end{array}$} & & \\
\hline$A$ & $\mathbf{v}$ & & $\mathbf{A}$ & $\mathrm{V}$ & & A & V & & $\mathbf{A}$ & V \\
\hline 44,5 & 42,1 & $-5,4$ & 924,0 & 896,0 & $-3,0$ & 39,93 & 35,59 & $-10,9$ & 539,19 & 511,25 \\
\hline$\$ 3,7$ & 42,4 & $-3,0$ & 946,4 & 925,4 & $-2,2$ & 33,85 & 32,12 & $-5,1$ & 558,13 & 537,29 \\
\hline 41,5 & 40,8 & $-1,7$ & 866,6 & 856,8 & $-1,1$ & 41,66 & 38,19 & $-8,3$ & 518,20 & 509,52 \\
\hline 45,0 & 43,5 & $-3,3$ & 1000,3 & 998,9 & $-0,1$ & 33,85 & 32,98 & $-2,6$ & 637,98 & 625,48 \\
\hline 45,1 & 42,7 & $-5,3$ & 1003,8 & 998,2 & $-0,5$ & 43,40 & 41,66 & $-4,0$ & 594,58 & 580,70 \\
\hline 42,9 & 41,0 & $-4,4$ & 1100,4 & 1064,0 & $-3, \mathbf{3}$ & 43,40 & 37,32 & $-14,0$ & 634,51 & 600,66 \\
\hline 46,4 & 44,2 & $-4,7$ & 897,4 & 888,3 & $-1,0$ & 40,80 & 38,19 & $-6,4$ & 552,04 & 541,03 \\
\hline 45,4 & 44,1 & $-2,9$ & 924,0 & 917,0 & $-0,7$ & 42,53 & 40,80 & $-4,1$ & 579,83 & 571,14 \\
\hline 41,3 & 40,1 & $-2,9$ & 985,6 & 959,0 & $-2,7$ & 34,72 & 32,98 & $-5,0$ & 605,86 & 578,96 \\
\hline 43,4 & 42,0 & $-3,2$ & 1059,8 & 1057,0 & $-0,3$ & 38,19 & 36,46 & $-4,5$ & 626,01 & 615,41 \\
\hline 46,4 & 44,2 & & 1100,4 & 1064,0 & & 43,40 & 41,66 & & 637,98 & 625,48 \\
\hline 41,3 & 40,1 & & 866,6 & 856,8 & & 33,85 & 32,12 & & 518,20 & 509,52 \\
\hline 43,9 & 42,3 & $-3,7$ & 970,47 & 956,06 & $-1,5$ & 39,23 & 36,63 & $-6,5$ & 584,63 & 567,14 \\
\hline
\end{tabular}

tritt des Blutes durch die Niere der Gesamt-N und der Rest- $\mathrm{N}$ ebenfalls und zwar der erstere eine geringe, der letztere eine etwas stärkere Abnahme, die beim ersteren um 1,5\% (0,1-3,3\%), beim letzteren um $6,5 \%(2,5-14,0 \%)$ stärker war als arterielle Werte. Albumin-N er. fuhr nach dem Durchtritt des Blutes durch die Niere ausnahmslos eine Abnahme (im Mittel 3,0\%), wohingegen der Globulin-N-Gehalt in sämtlichen Fällen im Nierenvenenblut eine Zunahme von durchschnittlich 1,5\% (0,4-3,6\%) aufwies. Mithin erfuhr der Albumin-GlobulinQuotient nach Nierendurchtritt des Blutes ausnahmslos eine prozentische Erniedrigung, die 1,2-6,4, im Mittel 4,1\% betrug. Serum-NaCl liess keine bedeutende Veränderung erkennen; da es indessen in den meisten Fällen (Versuch 2, 3, 4, 5, 6, 7 u. 10) im Nierenvenenblut ganz winzige Abnahme erfuhr, dürfte wohl wenigstens keine Zunahme resultiert haben. 
Tabelle 1. Fortsetzung.

\begin{tabular}{|c|c|c|c|c|c|c|c|c|c|}
\hline & \multicolumn{3}{|c|}{ Globulin-N } & \multicolumn{3}{|c|}{ Albumin / Globulin } & \multicolumn{3}{|c|}{ Serum-NaCl } \\
\hline \multirow{2}{*}{ 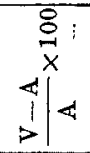 } & \multicolumn{2}{|c|}{$\mathrm{mg} / \mathrm{dl}$} & \multirow{2}{*}{$\begin{array}{c}8 \\
8 \\
\dot{x} \\
4 \mid \\
1 \mid\end{array}$} & \multirow{2}{*}{ A } & \multirow{2}{*}{ V } & \multirow{2}{*}{$\begin{array}{l}8 \\
x \\
1 \mid\end{array}$} & \multicolumn{2}{|c|}{$\mathrm{g} / \mathrm{dl}$} & \multirow{2}{*}{$\begin{array}{c}8 \\
-7 \\
x \\
1 \\
4 \mid\end{array}$} \\
\hline & $\mathbf{A}$ & V & & & & & $\mathbf{A}$ & $\mathrm{V}$ & \\
\hline$-5,2$ & 344,88 & 349,16 & $+1,2$ & 1,56 & 1,46 & $-6,4$ & 0,570 & 0,570 & \pm 0 \\
\hline$=3,7$ & 354,42 & 355,99 & $+0,4$ & 1,57 & 1,51 & $-3,8$ & 0,570 & 0,568 & $-0,5$ \\
\hline$-1,7$ & 306,74 & 309,09 & $+0,8$ & 1,67 & 1,65 & $-1,2$ & 0,560 & 0,555 & $-0,9$ \\
\hline$\therefore 1,9$ & 328,47 & 340,44 & $+3,6$ & 1,92 & 1,84 & $-4,2$ & 0,547 & 0,545 & $-0,4$ \\
\hline$-2,3$ & 365,82 & 375,84 & $+2,7$ & 1,62 & 1,54 & $-4,9$ & 0,605 & 0,600 & $-1,0$ \\
\hline$-5,3$ & 422,49 & 426,02 & $+0,8$ & 1,50 & 1,41 & $-6,0$ & 0,567 & 0,557 & $-1,7$ \\
\hline$-2 ; 0$ & 304,56 & 309,08 & $+1,5$ & 1,81 & 1,75 & $-3,3$ & 0,590 & 0,577 & $-2,2$ \\
\hline$-1,5$ & 301,64 & 305,06 & $+1,1$ & 1,92 & 1,87 & $-2,6$ & 0,535 & 0,535 & \pm 0 \\
\hline$\therefore 4,4$ & 345,02 & 347,06 & $+0,6$ & 1,76 & 1,67 & $-5,1$ & 0,550 & 0,550 & \pm 0 \\
\hline$-1,7$ & 395,78 & 405,13 & $+2,4$ & 1,58 & 1,52 & $-3,8$ & 0,590 & 0,580 & $-1,7$ \\
\hline & 422,49 & 426,02 & & 1,92 & 1,87 & & 0,605 & 0,600 & \\
\hline & 301,64 & 305,06 & & 1,50 & 1,41 & & 0,535 & 0,535 & \\
\hline$-3,0$ & 346,98 & 352,29 & $+1,5$ & 1,69 & 1,62 & $-4,1$ & 0,568 & 0,564 & $-0,8$ \\
\hline
\end{tabular}

Oben geschilderte Ergebnisse lassen sich dahin zusammenfassen: Aus der Tatsache, dass Hämoglobinwerte zur Hälfte der Fälle im Nierenvenenblut zunehmen, kann leicht darauf geschlossen werden, dass sich das Blut auf dem Wege des Durchtrittes durch die Niere eindickt, indem das im Blut enthaltene Wasser zum Teil hier ausgeschieden wird. Eiweisswerte nehmen hingegen in der Mehrzahl der Fälle (ausgenommen Versuch 4) nach Durchtritt des Blutes durch die Niere ab: Dieser Eiweissverlust dürfte wahrscheinlich darauf beruhen, dass das Serumeiweiss zum Teil bis zu seinen Endprodukten zersetzt und im Harn ausgeschieden worden ist. Dass die Herabsetzung des k. o. D. im Nierenvenenblut, welche auch von $P \mathrm{ein}^{14}$ und $\mathrm{Häb-}$ ler, Gǘn ther, Schein er, W a gner u. Wörner ${ }^{16)}$ konstatiert wúrde, durchaus nicht durch eine einfache Abnahme des Eiweisses verursacht ist, geht aus dem Umstand hervor, dass sich der Druck pro \% 
erniedrigt, ferner dass bei der Abnahme des Albumins das Globulin die Zunahme aufweist. In der Niere nämlich vollzieht sich ein besonderer Eiweissaustausch öder Eiwessumsatz, wobei das Bluteiweiss mehr nach grobdisperser Seite hin verschoben ist. T Tnaka ${ }^{13}$ hat darauf hingewiesen, dass sich in her Niere das Albumin ins Globulin umwandeln kann. Aber da in vorliegendem Versuch die prozentische Abnahme des Albumin- $\mathrm{N}$ mit prozentischer Zunahme des Globulins nicht gleichen Schritt hält, sondern die Abnahme des erstern im allgemeinen grösser ist; dürfte es sich hier höchstwahrscheinlich darum handeln, dass nicht nur ein Teil des Albumins ins Globulin umgewandelt wird, sondern auch dass sich die Spaltung des Eiweisses von Albumingruppe lebhafter abspielt und ein Teil desselben als Nicht-Eiweiss- $\mathrm{N}$ im Harn ausgeschieden wird.

Die auffallende Herabsetztung des Rest-N, welche nach Dürchtritt des Blutes durch die Niere unfehlbar eintritt, wird bekanntlich durch die wichtige Funktion der Niere bewerkstelligt. Das Serum$\mathrm{NaCl}$ dürfte deshalb abnehmen, weil es zum Teil beim Durchtritt durch die Niere samt dem Wasser ausgeschieden wird.

\section{Versuch am Urankaninchen.}

In vorliegender Versuchsreihe wurden 6 gesunde Kaninchen verwendet. Es wurden nämlich Versuchstieren $0,5 \mathrm{ccm}$ einer $5 \%$ igen Urannitratlösung pro kg Körpergewicht subkutan injiziert. Nachdem ca. 48 Stunden später Vergiftungserscheinungen deutlich aufgetreten waren, wurde mit dem Versuch begonnen. Da es sehr schwierig war, an ein und demselben Tier vor der Vergiftung aus der V. renalis und Arterie Blutproben zur Kontrolle zu entnehmen und dann nach erfolgter Vergiftung wieder die Blutentnahme vorzunehmen, und zumal aus Rücksicht auf etwaige Beeinflussung durch die Anämie wurde die Blutentnahme zur Kontrolle unterlassen. Die Befunde, welche nach dem Eintritt der Vergiftungssymptome erhoben wurden, wurden mit dem an gesunden Kaninchen erhaltenen Resultaten vergleichend betrachtet.

Die nach erfolgter Vergiftung erhaltenen Resultate sind in Táb. 2 wiedergegeben. Zum besseren Verständnis sind im unteren Teil der Tabelle normale Durchschnittswerte sowie prozentische Ab- und Zunahmen beim Urankaninchen, welche aus dem Vergleich mit normalen Durchschnittswerten resultieren, aufgezeichnet.

Zunächst seien hier nach der Vergiftung ermittelte Durchschnittswerte mit normalen Werten verglichen. Der Hämoglobin- und Eiweissgehalt zeigen im Arterienblut deutliche Abnahmen von 5,1\% und 8,0\% während beide im Nierenvenenblut noch ausgesprochene Abnah- 
Tabelle

Versuch an

\begin{tabular}{|c|c|c|c|c|c|c|c|c|}
\hline \multirow{3}{*}{$\begin{array}{l}\text { Versuchs- } \\
\text { nummer u. } \\
\text { Geschlecht }\end{array}$} & \multicolumn{2}{|c|}{$\begin{array}{c}\text { Körpergewlcht } \\
(\mathrm{kg})\end{array}$} & \multicolumn{3}{|c|}{ Hämoglobin } & \multicolumn{3}{|c|}{ Serumeiweiss } \\
\hline & \multirow{2}{*}{$\begin{array}{l}\text { vor } d . \\
\text { Vergif- } \\
\text { tung }\end{array}$} & \multirow{2}{*}{$\begin{array}{c}\text { beim } \\
\text { Versuch }\end{array}$} & \multicolumn{2}{|c|}{$\mathrm{g} / \mathrm{dl}$} & \multirow{2}{*}{$\begin{array}{c}\frac{8}{8} \\
\frac{4}{1} \\
\left.0\right|^{4}\end{array}$} & \multicolumn{2}{|c|}{$\%$} & \multirow{2}{*}{$\begin{array}{l}8 \\
8 \\
x \\
4 \mid \\
1 \\
1\end{array}$} \\
\hline & & & $\mathbf{A}$ & $\mathbf{V}$ & & A & $\mathbf{v}$ & \\
\hline $1 \delta$ & 2,30 & 2,20 & 13,47 & 13,47 & \pm 0 & 6,21 & 5,88 & $-5,3$ \\
\hline 2 古 & 2,00 & 1,89 & 14,05 & 14,05 & \pm 0 & 5,70 & 5,47 & $-4,0$ \\
\hline 3 t & 2,05 & 1,87 & 13,64 & 13,64 & \pm 0 & 6,10 & 5,83 & $-4,4$ \\
\hline $4 \hat{\mathrm{o}}$ & 1,98 & 1,90 & 14,24 & 14,24 & \pm 0 & 5,79 & 5,55 & $-4,1$ \\
\hline 58 & 2,13 & 2,00 & 13,22 & 13,22 & \pm 0 & 5,86 & 5,79 & $-1,2$ \\
\hline $6 \hat{8}$ & 1,90 & 1,81 & 14,80 & 14,80 & \pm 0 & 6,68 & 6,19 & $-7,3$ \\
\hline \multicolumn{3}{|c|}{ Durchschnitt } & 13,90 & 13,90 & \pm 0 & 6,06 & 5,78 & $-4,4$ \\
\hline \multicolumn{3}{|c|}{ Durchschnitt d. Normalwerte } & 14,65 & 14,73 & $+0,5$ & 6,59 & 6,47 & $-1,9$ \\
\hline \multicolumn{3}{|c|}{$\begin{array}{l}\text { Prozentische Ab-u. Zunahme } \\
\text { im Vergleich mit d. Norm }\end{array}$} & $-5,1$ & $-5,6$ & & $-8,0$ & $-10,7$ & \\
\hline
\end{tabular}

A : Arterienblut.

V: Nierenvenenblut.

men von 5,6\% und 10,7\% aufweisen. K. o. D. und Druck pro \% erfahren im Arterienblut gleich Abnahmen von 16,2\% und 8,4\%, im Venenblut auch Abnahmen von 17,9\% und 8,7\%. Bezüglich der Eiweissfraktionen erfahren der Gesamt- $\mathrm{N}$ und Rest-N sowohl im Arterienblut als auch im Nierenvenenblut ebenfalls stärkere Zunahmen als bei der Kontrolle, insbesondere die Zunahme des Rest- $\mathrm{N}$ ist auffallend. Albumin- und Globulin- $\mathrm{N}$ indessen nehmen im Arterienblut sowie im Venenblut gegenuiber der Norm ab und zwar der erstere in erheblichem Masse, der letztere in relativ geringem Masse. Wenn man diese prozentischen Abnahmen mit normalen Werten vergleicht, so erkennt man, dass beide im Venenblut gemeinsam stärkere Abnahmen aufweisen. Der Albumin-Globulin-Quotient erfäh"t gegenüber der Norm eine beträchtliche Verminderung; die prozentische Verminderung erweist sich aber als dieselbe im Arterienblut und Venenblut, sie beträgt nämlich in beiden Blutarten -13,0\%.

Vergleicht man die an Urankaninchen ermittelten Daten im Arterien- und Nierenvenenblut gegeneinander, so ergibt sich, dass der Hämoglobingehalt in keinem Fall nach Durchtritt des Blutes durch die 
2.

Urankaninchen.

\begin{tabular}{|c|c|c|c|c|c|c|c|c|}
\hline \multicolumn{3}{|c|}{ K. o. D. } & \multicolumn{3}{|c|}{ Druck pro\% } & \multicolumn{3}{|c|}{ Gesamt-N } \\
\hline \multicolumn{2}{|c|}{$\mathrm{mmH}_{2} \mathrm{O}$} & \multirow{2}{*}{$\begin{array}{l}\frac{8}{x} \\
1 \\
4 \\
4\end{array}$} & \multicolumn{2}{|c|}{$\mathrm{mmH}_{2} \mathrm{O}$} & \multirow{2}{*}{$\frac{8}{8}$} & \multicolumn{2}{|c|}{$\mathrm{mg} / \mathrm{dl}$} & \multirow{2}{*}{$\begin{array}{c}\frac{8}{x} \\
4 \\
1 \\
1\end{array} \mid$} \\
\hline $\mathbf{A}$ & $\mathbf{V}$ & & $\mathbf{A}$ & $\mathrm{V}$ & & A & V & \\
\hline 246 & 224 & $-8,9$ & 39,5 & 38,1 & $-3,5$ & 984,2 & 950,6 & $-3,4$ \\
\hline 239 & 224 & $-6,3$ & 42,0 & 40,9 & $-2,6$ & 967,4 & 946,4 & $-2,2$ \\
\hline 251 & 235 & $-6,4$ & 41,1 & 40,3 & $-1,9$ & 1061,2 & 1024,8 & $-3,4$ \\
\hline 232 & 210 & $-9,5$ & 40,1 & 37,8 & $-5,7$ & 1029,0 & 978,6 & $-4,9$ \\
\hline 242 & 232 & $-4,1$ & 41,3 & 40,1 & $-2,9$ & 963,2 & 938,0 & $-2,6$ \\
\hline 248 & 222 & $-10,5$ & 37,1 & 35,9 & $-3,2$ & 1118,6 & 1052,8 & $-5,9$ \\
\hline 243 & 224 & $-7,6$ & 40,2 & 38,8 & $-3,3$ & 1020,6 & 981,87 & $-3,7$ \\
\hline 290 & 273 & $-5,7$ & 43,9 & 42,5 & $-3,7$ & 970,47 & 956,06 & $-1,5$ \\
\hline$-16,2$ & $-17,9$ & & $-8,4$ & $-8,7$ & & $+5,2$ & $+2,7$ & \\
\hline
\end{tabular}

Niere vermehrt gefunden wird und dass zwischen dem arteriellen und venösen Werte kein Unterschied besteht. Serumeiweiss und k. o. D. zeigen im Nierenvenenblut im Vergleich zur Norm mehr oder weniger ausgesprochene Abnahmen, welche sich beim ersteren auf 4,4\% (1,2$7,3 \%)$, beim letzteren auf 7,6\% (4,1-10,5\%) beliefen. Der Unterschied des Drucks pro \% zwischen dem Arterien- und Venenblut verhält sich indessen beinahe analog wie bei gesunden Kaninchen. Was die Veränderungen an Fraktionen anbetrifft, erfahren der Gesamt-N und Albumin- $\mathrm{N}$ nach Nierenpassage des Blutes stärkere Abnahmen als bei gesunden Kaninchen, das Globulin-N nimmt, ausgenommen einen Fall (Versuch 2), im Gegensatz zu gesunden Kaninchen, im Nierenvenenblut ab. Der Albumin-Globulin-Quotient verhält sich, in Analogie zum Druck pro \% hinsichtlich der Beziehung zwischen dem Arterienund Venenblut auf eben gleiche Weise wie in der Norm. Allein der Rest-N zeigt nach Durchtritt des Blutes durch die Niere keine einheitlichen Werte; falls er abnimmt, tut es nur in äusserst geringem Masse, im ganzen genommen weist er im Nierenvenenblut eine geringfügige Zunahme auf.

Serum-NaCl bietet, dem Durchschnittswert nach, gegeniiber dem Kontrollwert eine geringe Zunahme dar, die Verhältnisse vor und nach 
Tabelle 2. Fortsetzung.

\begin{tabular}{|c|c|c|c|c|c|c|c|c|}
\hline \multicolumn{3}{|c|}{ Rest-N } & \multicolumn{3}{|c|}{ Albumin-N } & \multicolumn{3}{|c|}{ Globulin-N } \\
\hline \multicolumn{2}{|c|}{$\mathrm{mg} / \mathrm{dl}$} & \multirow{2}{*}{ 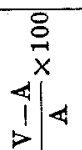 } & \multicolumn{2}{|c|}{$\mathrm{mg} / \mathrm{dl}$} & \multirow{2}{*}{$\begin{array}{l}\frac{8}{8} \\
x \\
\mid \\
\mid\end{array} \mid$} & \multicolumn{2}{|c|}{$\mathrm{mg} / \mathrm{dl}$} & \multirow{2}{*}{$\begin{array}{l}8 \\
\frac{8}{x} \\
1 \\
1 \\
\mid 1\end{array}$} \\
\hline A & V & & $\mathbf{A}$ & $\mathrm{V}$ & & $\mathbf{A}$ & $\mathbf{V}_{\text {: }}$ & \\
\hline 105,03 . & 103,29 & $-1,6$ & 525,14 & 500,84 & $-4,6$ & 354,03 & 346,47 & $-2,1$ \\
\hline 214,40 & 214,40 & \pm 0 & 441,68 & 419,24 & $-5,1$ & 311,32 & 312,76 & $+0,5$ \\
\hline 209,19 & 206,58 & $-1,2$ & 512,99 & 489,56 & $-4,6$ & 339,02 & 328,66 & $-3,0$ \\
\hline 244,78 & 244,78 & \pm 0 & 477,40 & 434,00 & $-9,1$ & 306,82 & 299,82 & $-2,3$ \\
\hline 138,14 & 141,48 & $+2,4$ & 499,84 & 474,80 & $-5,0$ & 325,22 & 321,72 & $-1,1$ \\
\hline 177,07 & 185,75 & $+4,9$ & 533,82 & 482,61 & $-9,6$ & 407,71 & 384,44 & $-5,7$ \\
\hline 181,43 & 182,71 & $+0,7$ & 498,48 & 466,84 & $-6,3$ & 340,69 & 332,31 & $-2,3$ \\
\hline 39,23 & 36,63 & $-6,5$ & 584,63 & 567,14 & $-3,0$ & 346,98 & 352,29 & $+1,5$ \\
\hline$+362,5$ & $+398,8$ & & $-14,7$ & $-17,7$ & & $-1,8$ & $-5,7$ & \\
\hline
\end{tabular}

dem Durchtritt durch die Niere zeigen keine bedeutsame Abweichung von der Kontrolle.

\section{Versuch an Kantharidinkaninchen.}

Zum vorliegenden Versuch wurden 5 Kaninchen eine 0,2\%ige Kantharidinessigätherlösung in Mengen von $0,5 \mathrm{ccm}$ pro kg Körpergewicht im Intervall von 24 Stunden zweimal subkutan injiziert. Nachdem 24 Stunden nach zweiter Injektion von dem Auftreten der Vergiftungserscheinungen, wie Hämaturie und Albumunurie, überzeugt worden war, wurde Vérsuch angestellt (Tab. 3).

Wenn man zunächst an Kantharidintieren ermittelte Werte mit an normalen Kaninchen erhaltenen Werten vergleicht, so ergibt sich folgendes: Bei erstgenannter Tiergruppe erfahren Hämoglobin, Serumeiweiss, k. o. D. sowie Druck pro \% sowohl im Arterienblut wie auch im Nierenvenenblut erhebliche Abnahmen, wobei die Abnahme des k. o. D. besonders ausgeprägt (ca. -16\%) ist. Die Abnahmen sind bei 3 erstgenannten Grössen gemeinschaftlich im Venenblut grösser als im Arterienblut. Was die Eiweissfraktionen betiifft, erfolgt die Abnahme des Globulin-N in ganz geringem Masse, wohingegen das. 


\begin{tabular}{|c|c|c|c|c|c|c|}
\hline \multicolumn{3}{|c|}{ Albumin / Globulin } & \multicolumn{3}{|c|}{ Serum-NaCl } & \multirow{3}{*}{ Bemerkung. } \\
\hline \multirow{2}{*}{ A } & \multirow{2}{*}{ V } & \multirow{2}{*}{$\begin{array}{l}8 \\
\bar{x} \\
4 \\
1 \\
1\end{array}$} & \multicolumn{2}{|c|}{$\mathrm{g} / \mathrm{dl}$} & \multirow{2}{*}{$\begin{array}{c}8 \\
\dot{8} \\
x \\
4 \\
1 \\
1\end{array}$} & \\
\hline & & & A & $\mathrm{v}$ & & \\
\hline 1,48 & 1,44 & $-2,7$ & 0,612 & 0,610 & $-0,3$ & Eiweiss im Harn $9 \%$ \\
\hline 1,42 & 1,34 & $-5,6$ & 0,555 & 0,555 & \pm 0 & Eiweiss im Harn 14\% \\
\hline 1,51 & 1,49 & $-1,3$ & 0,597 & 0,592 & $-0,8$ & Eiweiss im Harn $2 \%$ \\
\hline 1,56 & 1,45 & $-7,0$ & 0,577 & 0,565 & $-2,1$ & Eiweiss im Harn $10 \%$ \\
\hline 1,54 & 1,47 & $-4,5$ & 0,607 . & 0,605 & $-0,3$ & Eiweiss im Harn $4 \%$ \\
\hline $1 ; 31$ & 1,25 & $-4,6$ & 0,605 & 0,605 & \pm 0 & Eiweiss im Harn $7 \%$ \\
\hline 1,47 & 1,41 & $-4,3$ & 0,592 & 0,589 & $-0,6$ & \\
\hline 1,69 & 1,62 & $-4,1$ & 0,568 & 0,564 & $-0,8$ & \\
\hline$-13,0$ & $-13,0$ & & $+4,2$ & $+4,4$ & & \\
\hline
\end{tabular}

Albumin-N und der Albumin-Globulin-Quotient beträchtliche Abnahmen erfahren. Rest-N erfährt, in analoger Weise, wie bei der Uranvergiftung, auffallend stärkere Zunahme als bei gesunden Kaninchen.

Betreffend Werte, die am Kantharidintier vor und nach Durchtritt des Blutes durch die Niere ermittelt wurden, zeigten Hämoglobinwerte ähnlich wie bei der Uranvergiftung keinen Unterschied zwischen dem Arterien- und Venenblut. Serumeiweiss erfuhr in analoger Weise wie bei gesunden Kaninchen im Venenblut in allen Fällen eine Abnahme, deren Grad grösser als in der Norm war; sie betrug nämlich 3,5\% (2,0$5,6 \%$ ), während sie bei gesunden Kaninchen nur 1,9\% ausmachte. K. o. D. erfuhr auch im Venenblut eine Abnahme von durchschnittlich $6,6 \%(5,2-9,6 \%)$, welche etwas grösser als in der Norm war. Druck pro \% verhielt sich je nach einzelnen Fällen verschieden, im grossen ganzen aber stand $\frac{\mathrm{V}-\mathbf{A}}{\mathbf{A}} \times 100$ nahe an $\operatorname{der} \operatorname{Norm}(-3,3 \%)$. Gesamt-N und Albumin-N erfuhren nach Nierendurchstrom des Blutes grössere Abnahmen als in der Norm. Während beide Abnahmen bei gesunden Kăninchen gleich $1,5 \%$ und 3,0\% betrugen, beliefen sie sich hier auf $3,5 \%$ und $5,5 \%$. Globulin-N, welcher in der Norm im Venenblut zu- 
Tabelle

Versuch an Kantharidin

\begin{tabular}{|c|c|c|c|c|c|c|c|c|}
\hline \multirow{3}{*}{$\begin{array}{l}\text { Versuchs- } \\
\text { nummer u. } \\
\text { Geschlecht }\end{array}$} & \multicolumn{2}{|c|}{$\begin{array}{l}\text { Körpergewicht } \\
(\mathrm{kg}) \quad \text {. }\end{array}$} & \multicolumn{3}{|c|}{ Hämoglobin } & \multicolumn{3}{|c|}{ Serumeiweiss } \\
\hline & \multirow{2}{*}{$\begin{array}{l}\text { vor d. } \\
\text { Vergif- } \\
\text { tung }\end{array}$} & \multirow{2}{*}{$\begin{array}{c}\text { beim } \\
\text { Versuch }\end{array}$} & \multicolumn{2}{|c|}{$\mathrm{g} / \mathrm{dl}$} & \multirow{2}{*}{ 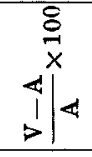 } & \multicolumn{2}{|c|}{$\%$} & \multirow{2}{*}{$\mid \begin{array}{c}8 \\
x \\
4 \\
1 \\
1 \\
>\end{array}$} \\
\hline & & & $\mathbf{A}$ & V & & $\mathbf{A}$ & $\mathrm{V}$ & \\
\hline $1 \delta$ & 1,80 & 1,69 & 13,08 & 13,08 & \pm 0 & 5,72 & 5,40 & $-5,6$ \\
\hline $2 \preccurlyeq$ & 2,00 & 1,92 & 13,36 & 13,36 & \pm 0 & 6,38 & 6,19 & $-\mathbf{3 , 0}$ \\
\hline $3 \hat{\delta}$ & 1,75 & 1,62 & 14,02 & 14,02 & \pm 0 & 5,88 & 5,75 & $-2,2$ \\
\hline $4 \delta$ & 1,77 & 1,70 & 13,47 & 13,47 & \pm 0 & 6,14 & 5,86 & $-4,6$ \\
\hline $5 \hat{\delta}$ & 1,92 & 1,92 & 14,24 & 14,24 & \pm 0 & 6,36 & 6,23 & $-2,0$ \\
\hline \multicolumn{3}{|c|}{ Durchschnitt } & 13,63 & 13,63 & \pm 0 & 6,10 & 5,89 & $-3,5$ \\
\hline \multicolumn{3}{|c|}{ Durchschnitt d. Normalwerte } & 14,65 & 14,73 & $+0,5$ & 6,59 & 6,47 & $-1,9$ \\
\hline \multicolumn{3}{|c|}{$\begin{array}{l}\text { Prozentische Ab-u. Zunahme } \\
\text { im Vergleich mit d. Norm }\end{array}$} & $-7,0$ & $-7,5$ & & $-7,4$ & $-9,0$ & \\
\hline
\end{tabular}

A: Arterienblut.

V: Nierenvenenblut.

nahm, erfuhr bei Kantharidintier in ähnlicher Weise, wie bei Urantier umgekehrt eine Abnahme von 1,6\%. Der Albumin-Globulin-Quotient erfuhr im Nierenvenenblut in allen Fällen eine Herabsetzung, die aber derselben bei gesunden Kaninchen fast gleichkam mit einem Betrag von $+4,0 \%(2,6-6,2 \%)$. Rest-N liess keine einheitliche Veränderung erkennen, zeigte aber im Durchschnitt eine geringe Abnahme (1,2\%).

Serum-NaCl wies im Durchschnittswert eine mässige Zunahme auf, die Quote vor und nach Durchtritt des Blutes durch die Niere war in ähnlicher Weise wie in der Norm im Venenblut um ein geringes herabgesetzt.

\section{Besprechung.}

Dass bei Funktionsschädigung der Niere der Eiweissgehalt des Serums abnimmt und diese Abnahme durch relative sowie absolute Verminderung der verhältnismässig kleineren Eiweissteilchen, wie der Albumingruppe bedingt ist, ist, wie eingangs bereits berührt, durch eine Reihe der Autoren von der Seite des k. o. D. und der Eiweissfraktionen erwiesen worden, und in guter Übereinstimmung damit stehen 
3.

kaninchen.

\begin{tabular}{|c|c|c|c|c|c|c|c|c|}
\hline \multicolumn{3}{|c|}{ IK. $\mathbf{~ . ~ D . ~}$} & \multicolumn{3}{|c|}{ Druck pro } & \multicolumn{3}{|c|}{ Gesamt-N } \\
\hline \multicolumn{2}{|c|}{$\mathrm{mmH}_{2} \mathrm{O}$} & \multirow{2}{*}{$\underbrace{\frac{8}{x}}_{1}$} & \multicolumn{2}{|c|}{$\mathrm{mmH}_{2} \mathrm{O}$} & \multirow{2}{*}{$\begin{array}{c}\frac{8}{x} \\
+\left.\right|_{1} ^{4}\end{array}$} & \multicolumn{2}{|c|}{$\mathrm{mg} / \mathrm{dl}$} & \multirow{2}{*}{ 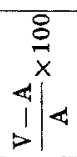 } \\
\hline $\mathbf{A}$ & V & & $\mathbf{A}$ & V & & A & V & \\
\hline 230 & 208 & $-9,6$ & 40,2 & 38,5 & $-4,2$ & 824,6 & 775,6 & $-5,9$ \\
\hline 250 & 235 & $-6,0$ & 39,2 & 38,0 & $-3,1$ & 980,0 & 946,4 & $-3,4$ \\
\hline 225 & 211 & $-6,2$ & 38,2 & 36,7 & $-3,9$ & 981,4 & 952,0 & $-3,0$ \\
\hline 248 & 233 & $-6,0$ & 40,4 & 39,8 & $-1,5$ & 953,4 & 917,0 & $-3,8$ \\
\hline 267 & 253 & $-5,2$ & 42,0 & 40,6 & $-3,3$ & 1023,4 & 1001,0 & $-2,2$ \\
\hline 244 & 228 & $-6,6$ & 40,0 & 38,7 & $-3,3$ & 952,56 & 918,40 & $-3,7$ \\
\hline 290 & 273 & $-5,7$ & 43,9 & 42,5 & $-3,7$ & 970,47 & 956,06 & $-1,5$ \\
\hline$-15,9$ & $-16,5$ & & $-8,9$ & $-8,9$ & & $-1,8$ & $-3,9$ & \\
\hline
\end{tabular}

auch die vorliegenden Versuchsergebnisse. Sowohl bei der Uranvergiftung wie auch bei der Kantharidinvergiftung finden wir nämlich eine erheblichere Abnahme des Serumeiweisses und ein stärkeres Absinken des k. o. D. als bei normalen Kaninchen. Mit den Fraktionen verhält es sich auch ähnlich; der Albumin-N und Globulin-N nehmen gleichfalls ab, die Abnahme des letzteren erweist sich indessen als äusserst geringfügig. Während diese Abnahme bei der Uranvergiftung im Arterienblut 1,8\%, im Venenblut 5,7\%, und bei der Kantharidinvergiftung gleich $0,1 \%$ und $3,1 \%$ beträgt, ist die Abnahme des Albumins bei beiden Vergiftungen viel grösser, indem sie sich bei Urantieren im Arterienblut auf 14,7\%, im Venenblut auf 17,7\% belief und bei Kanthridintieren im Arterienblut auf 12,2\%, im Venenblut auf $14,5 \%$ bezifferte. Aus alledem steht es also ausser allem $Z$ weifel, dass bei Nierenschädigungen, welcher Art sie auch sein mögen, das Bluteiweiss abnimmt, und diese Eiweissabnahme erfolgt offenbar vorwiegend auf Kosten feindisperser Eiweisskörper wie des Albumins.

Die Untersuchungen über das Verhalten des Eiweisses in dem der Niere zu- und dem davon abfliessenden Blut bei Nierenschädigungen sind, wie oben bereits angeführt, von Wiener ${ }^{12)}$ und Tanaka ${ }^{13)}$ ausgeführt worden. Nach Ergebnissen von Wien e ${ }^{12)}$ sollen im Nierenvenenblut Zunahmen des Gesamteiweisses und des Albumin-Globulin- 
Tabelle 3. Fortsetzung.

\begin{tabular}{|c|c|c|c|c|c|c|c|c|}
\hline \multicolumn{3}{|c|}{ Rest-N } & \multicolumn{3}{|c|}{ Albumin-N } & \multicolumn{3}{|c|}{ Globulin-N } \\
\hline \multicolumn{2}{|c|}{$\mathrm{mg} / \mathrm{dl}$} & \multirow{2}{*}{ 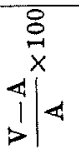 } & \multicolumn{2}{|c|}{$\mathrm{mg} / \mathrm{dl}$} & \multirow{2}{*}{$\begin{array}{l}8 \\
\stackrel{8}{1} \\
x \\
4 \mid 4 \\
1 \mid \\
1 \mid\end{array}$} & \multicolumn{2}{|c|}{$\mathrm{mg} / \mathrm{dl}$} & \multirow{2}{*}{$\begin{array}{c}8 \\
8 \\
x \\
4 \\
1 \\
1\end{array} \mid<$} \\
\hline A & $\mathrm{V}$ & & A & $\mathrm{v}$ & & A & $\mathrm{V}$ & \\
\hline 54,68 & 53,82 & $-1,6$ & 474,86 & 434,00 & $-8,6$ & 295,12 & 287,80 & $-2,5$ \\
\hline 70,31 & 68,57 & $-2,5$ & 537,29 & 508,65 & $-5,3$ & 372,40 & 369,18 & $-0,9$ \\
\hline 135,41 & 137,14 & $+1,3$ & 476,53 & 453,10 & $-4,7$ & 369,46 & 361,76 & $-2,1$ \\
\hline 85,93 & 83,33 & $-3,0$ & 526,01 & 499,97 & $-4,9$ & 341,46 & 333,70 & $-2,3$ \\
\hline 118,05 & 118,05 & \pm 0 & 550,31 & 528,61 & $-3,9$ & 355,04 & 354,34 & $-0,8$ \\
\hline 92,88 & 92,18 & $-1,2$ & 513,00 & 484,87 & $-5,5$ & 346,70 & 341,36 & $-1,6$ \\
\hline 39,23 & 36,63 & $-6,5$ & 584,63 & 567,14 & $-3,0$ & 346,985 & 352,29 & $+1,5$ \\
\hline 136,7 & $+151,6$ & & $-12,2$ & $-14,5$ & & $-0,1$ & $-3,1$ & \\
\hline
\end{tabular}

Quotienten aufgetreten sein, er hat aber Versuche nur an kleinen Material von 2 Uranhunden angestellt. Tan aka ${ }^{13)}$ hat Versuche an Uran- und Kantharidinhunden ausgeführt und kam zum Ergebnis, dass weder der Gesamt-N noch der Albumin-Globulin-Quotient nach Durchtritt des Blutes durch die Niere einheitliche Tendenz erkennen liess, indem beides zur Hälfte ab-, zur anderen Hälfte der Fälle zunahm. In vorliegenden Versuchen ist die Hämoglobinkonzentration sowohl bei Uran- wie auch bei Kantharidinvergiftung durch Durchtritt des Blutes durch dies Niere, im Gegensatz zu gesunden Kaninchen, keineswegs erhöht. Dies dürfte wahrscheinlich darauf zurückzuführen sein, dass die Wasserausscheidung durch die Nieren schlechter als in der Norm vor sich geht oder dass rote Blutzellen aus der Niere im Harn abgesondert werden oder beides zusammentrifft. Serumeiweiss erfährt bei beiden Vergiftungen, in analoger Weise wie bei gesunden Kaninchen, nach Nierenpassage des Blutes gemeinsame Abnahmen, deren Grad ist in einzelnen Fällen meistens grösser als in der Norm, wobei mithin auch im ganzen genommen grössere prozentische Abnahme resultiert. Wenn man nun diese Verhältnisse vom Standpunkte des Drucks pro \% und des Albumin-Globulin-Quotienten betrachtet, so springt folgende Tatsache in die Augen: Während $\frac{\mathrm{V}-\mathrm{A}}{\mathrm{A}} \times 100$ beider Daten bei gesunden Kaninchen gleich $-3,7 \%$ und $-4,1 \%$ beträgt, beläuft sie bei der 


\begin{tabular}{|c|c|c|c|c|c|c|}
\hline \multicolumn{3}{|c|}{ Albumin / Globulin } & \multicolumn{3}{|c|}{ Serum-NaCl } & \multirow{3}{*}{ Bemerkung } \\
\hline \multirow{2}{*}{ A. } & \multirow{2}{*}{$\mathrm{V}$} & \multirow{2}{*}{ 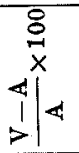 } & \multicolumn{2}{|c|}{$\mathrm{g} / \mathrm{dl}$} & \multirow{2}{*}{ 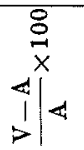 } & \\
\hline & & & $\mathbf{A}$ & $\mathrm{V}$ & & \\
\hline 1,61 & 1,51 & $-6,2$ & 0,610 & 0,600 & $-1,6$ & $\begin{array}{l}\text { Im Harn Eiweiss 5\%, } \\
\text { Erythrozyten spärlich. }\end{array}$ \\
\hline 1,44 & 1,38 & $-4,2$ & 0,595 & 0,590 & $-0,8$ & Hochgradige Hämaturie. \\
\hline 1,29 & 1,25 & $-3,1$ & 0,605 & 0,600 & $-0,8$ & $\begin{array}{l}\text { Im Harn Eiweiss } 2 \%, \\
\text { Erythrozyten reichlich. }\end{array}$ \\
\hline 1,54 & 1,50 & $-2,6$ & 0,585 & 0,585 & \pm 0 & $\begin{array}{l}\text { Im Harn Eiweiss } 1 \% o \\
\text { Erythrozyten mässig. }\end{array}$ \\
\hline 1,55 & 1,49 & $-3,9$ & 0,542 & 0,540 & $-0,4$ & $\begin{array}{c}\text { Im Harn Eiweiss } 2 \%, \\
\text { Erythrozyten mässig. }\end{array}$ \\
\hline 1,49 & 1,43 & $-4,0$ & 0,587 & 0,583 & $-0,7$ & \\
\hline 1,69 & 1,62 & $-4,1$ & 0,568 & 0,564 & $-0,8$ & \\
\hline$-11,8$ & $-11,7$ & & $+3,3$ & $+3,4$ & & \\
\hline
\end{tabular}

Uranvergiftuhg gleich auf $-3,3 \%$ und $-4,3 \%$, bei der Kantharidinvergiftung $-3,3 \%$ und $-4,0 \%$; ganz allgemein lässt sich also sagen, dass in bezug auf den Eiweissquotienten fast kein Unterschied zwischen der normalen und der geschädigten Niere besteht, mit anderen Worten, das Blut verliert durch Durchtritt durch die geschädigte Niere Albumin und Globulin in beinahe gleichen Mengen. Nach allem scheint die Auffassung wohl zu Recht zu bestehen, dass bei der. Uranvergiftung wie auch bei der Kantharidin vergiftung das Serumeiweiss durch Durchtritt durch die Niere quantitativ in höherem Masse als in der Norm vermindert ist, während in qualitativer Hinsicht Albumin und Globulin in annähernd gleichem Verhältnis abnehmen. Es erhebt sich nun die Frage, was für Schicksal erfahren die dieser Verminderung entsprechende Eiweissmenge. Man kann sich zwar zuerst vorstellen, dass derartiges Eiweiss ein wichtiges Material für die Albuminurie abgeben könne. Aber dass der Mechanismus hierfür sich doch keineswegs so einfach, sondern ausserordentlich kompliziert gestaltet, geht aus der Tatsache klar hervor, dass über die Frage nach der Genese der Albuminurie noch keine Einigung erzielt worden ist. Hewitt, ${ }^{20)} \mathrm{Wid}$ dowson, ${ }^{21)}$ Kozawa, Iwatsuru u. Adachi ${ }^{22)}$ u. a. vertreten z. B.

20) Hewitt, Biochem. Ztschr., 1927, 21, 216 n. 1109.

21) Wid dow s o n, Tbid., 1933. 27, 1321.

22) Kozawa, Iwatsuru u. Adachi, Biochem. Ztschr., 1933, 260, 313. 
den Standpunkt, dass das Eiweiss im Harn bei Nierenerkrankungen aus Blutproteinen, welche als solche abfiltriert und nicht irgend wie in der Niere verändert werden, stamme. Nach Schenck u. Schlü̈ter ${ }^{23 \lambda}$ u. a. soll dieStruktur des Harneiweisses von derselben des Bluteiweisses verschieden und deshalb das Harneiweiss durchaus nicht ein blosser, durch die Filtration des Bluteiwisses entstehendes Stoff sein.

Die Untersuchungen über das Harneiweiss sind von alters her ausgeführt worden und derzeitig ist es allgemein anerkannt worden, dass von den im Harn erscheinenden Eiweissarten meistenfalls das Albumin überwiegt. Bei der Durchsicht einschlägiger Literatur fällt uns jedoch gelegentlich die Angabe über das Überwiegen des Globulins über das Albumin auf. Seit Mitteilungen von Joachim, ${ }^{24)} \mathrm{Zack} \mathrm{u}$. Nec ker ${ }^{25}$ haben wir nämlich öfters von häufigerem Vorkommen der Globulinurie bei der Amyloidnephrose gehört, bei Nephritis hat $\mathrm{Cl}$ o ët ta ${ }^{26)}$ die Beobachtung gemacht, dass bei chronischer Form im Harneiweiss weniger Globulin vorkam, bei akuter Form aber der Albumin-GlobulinQuotient relativ niedrig war. Gross ${ }^{27)}$ Kollert u. Starlinger ${ }^{281}$ haben darüber berichtet, dass es Fälle gäbe, in denen die Globulinausscheidung die Albuminmenge übertrifft. Geill ${ }^{29}$ hat auch bei akuter Nephritis das Harneiweiss untersucht und fand, dass die Albuminmenge 65-70\% betrug.

Derartige Befunde gehören indessen meines Erachtens unter Besonderheiten, es ist allerdings eine von vielen Autoren anerkannte Tatsache, dass der Albumin-Globulin-Quotient des Harneiweisses hohe Werte von 5-10 aufweist.

Aus dieser Überlegung wäre es nicht angängig, aus der in annähernd gleichem Verhältnis erfolgten Verminderung von Albumin und Globulin, wie sie in vorliegendem Versuch auftrat, ohne weiteres darauf schliessen zu wollen, dass diese verminderte Eiweissmenge als solche als Harneiweiss eliminiert sei. In jüngster Zeit hat S a n a d a ${ }^{2)}$ an hiesiger Klinik an normal und nierengeschädigten Kaninchen Bestimmungen von Gewebseiweiss und dessen k. o. D. ausgeführt und kam zum Ergebnis, dass bei Uran- sowie Kantharidinkaninchen in der Niere sich enorme Abnahme des Gewebseiweisses, erhebliche $\mathrm{Zu}$ nahme des Rest-N und geringe Steigerung des Drucks pro \% einstellen; auf Grund dieses Ergebnisses ist er der Meinung, dass das Gewebseiweiss in der Niere unter der Giftwirkung sich spaltet, verkleinert und

23) Schenck u. S chlüter, Arch. f. exp. Pathol. u. Pharmak., 1933, 169, 343.

24) Joa chim, Arch. f. d. ges. Physiol., 1903, 93, 558.

25) Za ck u. Necker, Dtsch. Arch. f. klin. Med., 1907, 88, 542.

26) Clo ëtta, Arch. f. exp. Pathol. u. Pharmak., 1899, 42, 453.

27) Gross, Dtsch. Arch. f. klin. Med., 1906, 186, 578.

28) Kollert u. Starlinger, Ztschr. f. klin. Med., 1926, 104, 44.

29) Geill, Tbid., 1929, 110, 334. 
so zum Teil aus der Niere ausgeschieden werden dürfte. Betrachtet man nun im Zusammenhang mit dieser Angabe die Ergebnisse vorliegenden Versuchs, so wäre die Annahme wohl zutreffend, dass das Harneiweiss aus dem Serumeiweiss und dem in der Niere verkleinerten Gewebseiweiss entsteht.

Allgemein bekannt ist die Vermehrung von Rest- $\mathrm{N}$ im Blut bei Nierenerkrankungen, auch in vorliegendem Versuch trat die Zunahme des Rest-N bei beiden Vergiftungen, besonders aber bei der Uranvergiftung ausgesprochen in Erscheinung. Die Ursache für die Rest-NErhöhung bildet allerdings die erhebliche Zersetzung des Blut- und Gewebseiweisses durch Vergiftungen; über den Mechanismus der Rest$\mathrm{N}$-Anhäufung im Blut sind indessen dieMeinungen sehr geteilt. Nach Anschauungen von einer Reihe Autoren vollzieht sich die Anhäufung von Rest-N zuerst in den Geweben, und dann im Blut (Rose mann, ${ }^{30 \text { ) }}$ Monakow $\left.{ }^{31}\right)$, andere Autoren behaupten demgegenüber initiale Anhäufung im Blut (Rosenberg, ${ }^{32)}$ Rohony u. Lax ${ }^{33)}$ ) und wieder andere nehmen gleichzeitige Anhäufung des Rest-N in Blıt und Geweben an (Soetbeer u. Schmidt, ${ }^{34)}$ Marshall u. Davis, ${ }^{35)}$ Weiss u. Garner ${ }^{36)}$ u. a.). Jedenfalls ist die wesentliche Ursache in Ausscheidungsstörungen der Niere zu suchen. Im Kontrollversuch vorliegender Untersuchung wurde stets eine Herabsetzung des Rest-N im Nierenvenenblut konstatiert, während bei Nierenschädigungen der im Arterienblut angehäufte Rest- $\mathrm{N}$ nach Durchtritt desselben durch die Niere nur im geringen Masse oder gar nicht abnahm, ja sogar mitunter eher zunahm. Bei der Uranvergiftung trat diese Tendenz besonders augenscheinlich in den Vordergrund. Es ist dies zum Teil auf die vermehrte Bildung von Rest- $\mathrm{N}$ in der Niere selbst, aber hauptsächlich auf die Störung des Ausscheidungsvermögens der Niere zurückzuführen. Nach alledem wäre die Auffassung naheliegend, dass der Rest- $\mathrm{N}$, der bei Nierenschädigungen im Körper vermehrt gebildet ist, schwer ausgeschieden wird, so dass es zur Anhäufung des Rest- $\mathrm{N}$ kommt und dieser angehäufte Rest-N sich als Gift auswirkt, indem er die Zersetzung des Körpereiweisses immer intensiver bewirkt, wobei es zum Cir-

30) R o se m a n n, Pflüger's Arch., 1904, 72, 467.

31) Monakow, Dtsch. Arch. klin. Med., 1914, 116, 1. u. 1917, 122, 241.

32) Rosen berg, Arch. f. exp. Path. u. Pharmak., 1920, 87, 86. u. 153.

33) Rohony u. Lax, Ztschr. f. klin. Med., 1922, 93, 217.

34) Soetbeer u. Schmidt, Zit nach Strauss: Die Nephritiden, 3. Aufl., Berlin, 1920, 143.

35) Marshall u. Devis, Journ. Biol. Chem., 1914, 18, 53.

36) We is s. Garner, Journ. Lab. \& Clin. Med., 1921-22, 7, 229. 
culus vitiosus und folglich zu immer erheblicherer Rest-N-Erhöhung im Blut kommt.

\section{Zusammenfassung.}

Es wurden an normalen Kaninchen sowie Uran- und Kantahridinkaninchen Untersuchungen über quantitative sowie qualitative Veränderungen der Bluteiweisskörper vor und nach dem Durchtritt des Blutes durch die Niere ausgeführt, wobei auch zugleich vergleichende Bestimmungen von Hämoglobin und Serum-NaCl ausgeführt wurden. Diese Ergebnisse sind im folgenden kurz zusammenfasst wiedergegeben.

1. Bei normalen Kaninchen : Serumeiweiss, kolloid-osmotischer Druck (k. o. D.) des Blutes sowie kolloid-osmotischer Druck pro \% Eiweiss (Druck pro \%) zeigen ausnahmslos niedriegere Werte im Nierenvenenblut als im Arterienblut, in Parallele damit erfahren Gesamt-N und Albumin-N Abnahmen. Globulin-N erfährt hingegen im Nierenvenenblut eine geringe Vermehrung. Der Albumin-GlobulinQuotient ist mithin nach dem Durchtritt des Blutes durch die Niere stets herabgesetzt. Rest-N zeigt im Nierenvenenblut auffallend niedrige Werte. Hämoglobin zeigt im Venenblut die Tendenz zu wenn auch geringfügiger Vermerhrung, wohingegen Serum- $\mathrm{NaCl}$ in den meisten Fällen abnimmt.

2. Bei Uran- und Kantharidinkaninchen: Die Abnahme des Serumeiweisses im Nierenvenenblut ist grösser als bei normalen Kaninchen, dementsprechend sinkt auch der k. o. D. ab, der Druck pro $\%$ verhält sich aber annähernd ähnlich wie in der Norm. Die Verminderungen von Gesamt- $\mathrm{N}$ und Albumin- $\mathrm{N}$ nach dem Durchtritt des Blutes durch die Niere sind auch grösser als in der Norm, Globulin-N ist durchaus nicht vermehrt, sondern herabgesetzt. Der AlbuminGlobulin-Quotient erfährt auch eine Erniedrigung, aber in annähernd gleichem Masse wie in der Norm. Rest-N nimmt im Nierenvenenblut, wenn auch um ein geringes, zu. Hämoglobin wird nach dem Durchtritt des Blutes durch die Niere in keinem Fall vermehrt gefunden. Das Verhalten des Serum-NaCl vor und nach dem Durchtritt durch die Niere erweist sich beinahe analog wie in der Norm, indem dasselbe im Venenblut in der Mehrzahl der Fälle abnimmt. 